\title{
Theoretical Chemistry and the Calculation of the Atmospheric State
}

\author{
Adrian F. Tuck (1D)
}

check for

updates

Citation: Tuck, A.F. Theoretical Chemistry and the Calculation of the Atmospheric State. Atmosphere 2021, 12, 727. https://doi.org/10.3390/ atmos12060727

Academic Editor: Luís Pedro Viegas

Received: 19 May 2021

Accepted: 4 June 2021

Published: 6 June 2021

Publisher's Note: MDPI stays neutral with regard to jurisdictional claims in published maps and institutional affiliations.

Copyright: (C) 2021 by the author. Licensee MDPI, Basel, Switzerland. This article is an open access article distributed under the terms and conditions of the Creative Commons Attribution (CC BY) license (https:// creativecommons.org/licenses/by/ $4.0 /)$.
Physics Department, Imperial College London, London SW7 2AZ, UK; adrianftuck@gmail.com

\begin{abstract}
Theoretical chemists have been actively engaged for some time in processes such as ozone photodissociation, overtone photodissociation in nitric acid, pernitric acid, sulphuric acid, clusters and in small organic acids. The last of these have shown very different behaviours in the gas phase, liquid phase and importantly at the air-water interface in aqueous aerosols. The founder of molecular dynamics, B J Alder, pointed out long ago that hydrodynamic behaviour emerged when the symmetry of a random, thermalised population of hard spheres—billiard balls—was broken by a flux of energetic molecules. Despite this, efforts over two centuries to solve turbulence by finding top-down solutions to the Navier-Stokes equation have failed. It is time for theoretical chemistry to try a bottom-up solution. Gibbs free energy that drives the circulation arises from the entropy difference between the incoming low-entropy beam of visible and ultraviolet photons and the outgoing higher-entropy flux of infrared photons over the whole $4 \pi$ solid angle. The role of the most energetic molecules with the highest velocities will affect the rovibrational line shapes of water, carbon dioxide and ozone in the far wings, where there is the largest effect on radiative transfer and hence on calculations of atmospheric temperature. The atmospheric state is determined by the interaction of radiation, chemistry and fluid dynamics on the microscopic scale, with propagation through the mesoscale to the macroscale. It will take theoretical chemistry to simulate that accurately. A challenging programme of research for theoretical chemistry is proposed, involving ab initio simulation by molecular dynamics of an air volume, starting in the upper stratosphere. The aim is to obtain scaling exponents for turbulence, providing a physical method for upscaling in numerical models. Turbulence affects chemistry, radiation and fluid dynamics at a fundamental, molecular level and is thus of basic concern to theoretical chemistry as it applies to the atmosphere, which consists of molecules in motion.
\end{abstract}

Keywords: molecular dynamics; stratospheric state; turbulence

\section{Introduction}

Atmospheric composition is determined by the interaction of radiation, fluid dynamics and chemistry. The calculation of these processes and their interactions is the province of theoretical chemistry, inspired by physical chemistry experiments, covering transitions through the scales from the microscale $\rightarrow$ mesoscale $\rightarrow$ macroscale. However, the 15 orders of magnitude in scale between the mean free path at surface pressure and a great circle in the atmosphere means that severe approximations and parametrisations have to be made in numerical modelling of atmospheric composition. Analytical solutions of the Navier-Stokes equation for fluid dynamics have not been found in two centuries, and consequently there is no rigorous mathematical framework to address the central issue of atmospheric turbulence. However, the emergence by symmetry breaking of hydrodynamic behaviour in a population of thermalised Maxwellian billiards subject to an organised flux [1,2] offers an approach from the smallest scales up, in contrast to the top-down methods used in atmospheric modelling, with its concomitant need to parametrise the lowest 8-10 orders of magnitude in scale. Until recently, the computational demands for an Alder-Wainwright approach have been insuperable, but it may be that it can now be essayed for the lowest three orders of magnitude. That would be sufficient to produce and 
examine scale invariance and the associated ability to incorporate Gibbs free energy [3-8] in order to diagnose directionality in the open, non-equilibrium system that is the atmosphere. Issues of dynamical phase transitions, mesoscale transitions [9], symmetry breaking, nonergodicity and frustration could also be addressed in principle by the occurrence of changes of slope in the diagnostic log-log plots [7]. For the underlying equations, [5] should be consulted.

The atmosphere consists of molecules in motion and given the driving importance of radiative transfers of photons from the ultraviolet to the far infrared, the chemistry affecting the abundance of the photon absorbing and emitting molecules is indissolubly linked to the fluid dynamics. The abundances of the radiatively active molecules fluctuate under the influence of the scale invariant turbulence, ensuring that on all scales from the mean free path to a great circle, some 15 orders of magnitude, fluid dynamics cannot be separated from radiation and chemistry. It is necessary to have at least three orders of magnitude to establish scaling; it is suggested that the lowest three orders up from the molecular mean free path in the upper stratosphere should constitute the initial attempt.

\section{Fluid Dynamics: Scale Invariance}

Alder and Wainwright [1] invented molecular dynamics by integrating the equations of motion for 220 hard spheres-'billiards'. They used the approach to computationally verify the Maxwell-Boltzmann distribution of atomic and molecular velocities, and the associated definition of temperature. They further established the emergence of fluid flow when the symmetry of a thermalised population of billiards was broken by the impingement of a flux of energetic molecules [2]. It was later shown, with the availability of much greater computer power, that the hydrodynamic flow of such a population showed scaling properties; it was scale invariant. Scale invariance has been demonstrated in observations of stratospheric chemistry [3-5], using the techniques pioneered by Schertzer and Lovejoy [6], and by Lovejoy and Schertzer [7]. Physical and theoretical chemical approaches were appealed for [3-5].

Some central questions arise at the transition from the microscale to the mesoscale. With sufficient computer power, it may be possible to replace billiards with more realistic representations of atmospheric molecules, and their collisions, including their roles in determining the line shapes of radiatively active transitions, in chemical reactions, in maintaining an operational temperature and in inducing hydrodynamic flow in what is an open system far from equilibrium. Scaling behaviour has been seen in atomistic dynamics calculations [8]. It would be of great interest to move towards a molecular dynamics simulation of an air volume including the essential radiative, dynamical and chemical processes. If successful it could address such questions such as the possible existence of dynamical phase transitions en route to the macroscale from the microscale via the mesoscale [9]. Important issues here are the role and representation of dissipation by radiation to space, and the translational and rotational population distributions of all air molecules, including $\mathrm{N}_{2}$ and $\mathrm{O}_{2}$. The scale and boundary conditions of such a simulated air packet would need careful consideration. The gap between its scale and the smallest available in suitable atmospheric observations would likely be 5-8 orders of magnitude [3]. Is there a dynamical phase transition in the upscaling to vorticity and potential vorticity when dissipation is included $[3,10,11]$ ? Ultimately, the formation of clusters and aerosol particles could, ambitiously, be considered, along with their radiative and chemical roles. The statistical multifractal equations by which the observed stratospheric-scale invariance is underpinned have been given elsewhere [3-5,12]. The driving force for the atmospheric circulation, the Gibbs free energy calculated as in [12] for the open non-equilibrium system, can be compared with that computed by conventional quantum statistical thermodynamic methods as though it was a closed system at equilibrium.

The equations for determining scale invariance and statistical multifractality are given in [5], where the variables are defined. Table 1 summarises the resulting equivalences 
between the variables of equilibrium quantum statistics and those deduced from statistical multifractal analysis of observations of the non-equilibrium atmosphere.

Table 1. Equivalence between statistical thermodynamic and scaling variables.

\begin{tabular}{ccc}
\hline Variable & Statistical Thermodynamics & Scaling Equivalent \\
\hline Temperature & $T$ & $1 / q k_{\text {Boltzmann }}$ \\
Partition function & $f$ & $\mathrm{e}^{-K(q)}$ \\
Energy & $E$ & $\gamma$ \\
Entropy & $-S(E)$ & $c(\gamma)$ \\
Gibbs free energy & $-G$ & $K(q) / q$ \\
\hline
\end{tabular}

It should be noted that the equivalences in Table 1 result from mappings, and are not merely formal similarities [5,7].

The starting variable for the analysis is $q$, defining the $q$ th order structure function of the observed variable under study. A log-log plot of the signal fluctuations versus its range enables derivation of the scaling exponent $K(q)$ and the Hurst exponent $H$, see [5]. Examination of energy $E$ in terms of a scale ratio results in an expression for the fractal co-dimension $c(\gamma)$. The mappings between the equilibrium quantum statistical thermodynamic variables in column 1 and their non-equilibrium scaling equivalents in column 2 are derived fully elsewhere $[5,7]$.

The Hurst exponent $H$, the intermittency $C_{1}$ and the Lévy exponent $\alpha$ are the scaling exponents that comprise the statistical multifractal description of atmospheric variability [3-5]. They offer insight into the chemical processes observed in the stratosphere; it will be of interest if theoretical chemical and molecular dynamics calculation can reproduce the observed exponents, justifying their interpretation [5]. More detailed physical explanations can be found in $[3-5,12]$. Later, the limiting effects of scale invariance, turbulence, symmetry breaking, emergence, non-ergodicity and frustration upon the applicability of the law of mass action in atmospheric chemistry and numerical models will be examined. Turbulence is ubiquitous in the atmosphere, and the aim must be to achieve a framework for its emergence based on molecular dynamics. It imposes its variability and intermittency on all molecules, so affecting chemistry and radiative transfer as well as the wind field. Turbulence is that property of emergent hydrodynamic behaviour that maximises the dilution of energy density, consistent with the minimisation of the Gibbs free energy based on scale invariance $[5,12]$.

\section{Theoretical Chemistry: Interpretation of Laboratory Experiments}

It has long been established that the state of the atmosphere is determined by the interaction of radiation, fluid dynamics and chemistry. In turn, this has led to an interplay between laboratory physical chemistry, radiative transfer as mediated by molecular spectroscopy, and numerical modelling of air flow; such is necessary to run computer simulations of the evolving state of the atmosphere. In Section 2, it was implicit that the air was simply a molecular gas. In practice, water vapour can form dimers and higher clusters. It also condenses on aerosols acting as condensation nuclei to form clouds. The aerosols themselves have compositions influenced by condensable vapours such as sulphuric and nitric acids. The clusters and aerosols have effects on both radiative transfer and chemistry. Theoretical chemistry has played a significant role in interpreting the processes observed in the laboratory that occur in the symmetry breaking environment of the air-water interface, particularly on aerosols.

\subsection{Atmospheric Aerosols and Clusters}

Atmospheric aerosols have become an important focal point during the last three decades, from both chemical and radiative perspectives. The development of laser mass spectrometry of individual atmospheric aerosol particles [13-15] revolutionised the study of their structural, chemical and radiative properties. High percentages of organic molecules 
were seen, extending through the upper troposphere and even into the lower stratosphere at lower, $5-10 \%$ levels. Those results led to the formulation of an inverted micelle model, with a liquid, aqueous sulphuric acid core encased in an external film of amphiphilic molecules [16]. This was followed by physicochemical laboratory experimentation which revealed a richly complex photo and surface chemistry of small organic acids, pioneered by the Vaida group and involving significant participation by theoretical chemists, selected examples of which are in [17-34]. There are one or more theoretical chemists involved in the authorship of each of these papers, who have deployed a selection of methods to interpret and understand the laboratory results. It is beyond the scope of this article to examine the theoretical approaches in detail. The point is that theoretical chemistry was essential for understanding these processes and their application to the atmosphere.

Pyruvic acid, for example, has shown diverse behaviours in solution, in the gas phase and at the air-water interface. Larger organic molecules have also been investigatedpeptide formation at the interface has been demonstrated, and the roles of phenylalanine and zymonic acid have been explored. Spontaneous photochemical generation of inverted micelles was seen, the role of chattering in line shapes was interpreted, and the partitioning between enol and keto forms observed and theoretically explained. The surface and interfacial processes on aerosols have been the subject of joint work by physical and theoretical chemists [31-39], for example.

Recently, scale invariance has been proposed as a means of calculating the Gibbs free energy and hence directionality in open, non-equilibrium systems [5], with particular reference to reactions in and on microdroplets-a category including atmospheric aerosols [40]. Experimental work has shown that this is a relevant subject [41-46]. Aerosols have also been proposed in the ancient atmosphere, as prebiotic chemical reactors [47-50]. The free radicals $\mathrm{OH}$ and $\mathrm{HO}_{2}$ would then, as now, have been agents of natural selection in the evolution of both interior and exterior chemistry of airborne bacteria and viruses.

Clusters, ranging from dimers to dimensions approaching the size where thermodynamics is applicable, have also been investigated, both experimentally [51-53] and theoretically [54,55], with consequent application to the atmosphere $[19,56]$, as regards both chemical and radiative effects. Chemical effects can encompass catalysis by water clustering on reactants, and also blocking [22,23,56]. Radiative effects can be direct via overtone absorption and indirect via the results of chemical transformations involving water-catalysed reactions.

\subsection{Photodissociation Processes}

Photodissociation of oxygen and ozone is the fundamental driver of atmospheric chemistry and is the process behind the production of the chain-carrying free radicals- $\mathrm{H}$, $\mathrm{OH}, \mathrm{HO}_{2}, \mathrm{NO}, \mathrm{NO}_{2}, \mathrm{Cl}, \mathrm{ClO}$ - that limit the production of odd oxygen, $\mathrm{O}\left({ }^{3} \mathrm{P}\right)+\mathrm{O}\left({ }^{1} \mathrm{D}\right)+$ $\mathrm{O}_{3}$. Those processes have been reviewed in many places elsewhere; here, we focus on what was a rather unexpected role for overtone photodissociation [57].

Overtone photochemistry was seen in the organic acids, following its laboratory observation. That followed from its observation in $\mathrm{HNO}_{3}, \mathrm{HO}_{2} \mathrm{NO}_{2}$ and $\mathrm{H}_{2} \mathrm{SO}_{4}$ and inclusion in modelling of atmospheric photochemistry, where it had significant impacts at low solar zenith angles and, in the case of sulphuric acid, in the upper stratosphere. Pernitric acid overtone photodissociation resolved what had been a persistent anomaly in the distribution of reactive odd nitrogen throughout the lower stratosphere. Theoretical chemists were involved in the calculation of these overtone photodissociation rates.

\section{Line Shapes and Radiative Transfer}

The line shapes of the rovibrational transitions of water vapour, carbon dioxide, ozone, nitrous oxide, methane, halocarbons and water clusters, including the dimer, have been measured by physical chemists with varying spectroscopic techniques. The data are fitted and used in retrieving abundances from satellite observations [51-53], in calculations of radiative transfer, and of the effect of water dimers and clusters [54] and thereby com- 
puting temperature profiles for the atmosphere [52]. That approach assumes that line shapes obtained from laboratory spectroscopy, which use equilibrium data, are directly applicable in the atmosphere. However, it has been observed in the lower stratosphere that correlations exist between temperature intermittency and the ozone photodissociation rate $[3-5,12]$, a result attributed to high-velocity excited photofragments transferring their translational energy to produce unthermalised $\mathrm{O}_{2}$ and $\mathrm{N}_{2}$ molecules. Theoretical chemistry calculations [58,59] played an essential role in this result. Calculations of line shapes based on laboratory observations and collisional theory produce symmetric line shapes, Doppler at low pressures, Lorentzian at high pressures and the convolution of the two in the Voigt shape in intermediate conditions [60]. Because the stronger lines of the absorbing and emitting molecules are self-absorbed near the line centres, the far wings of the lines play an important role in calculating atmospheric temperature, particularly in the case of future elevated levels of carbon dioxide. That is where the line shapes are most affected by the most energetic collisions between the molecules with the highest translational energies. If, as suggested by the correlation between ozone photodissociation rate and the intermittency of temperature, a Boltzmann distribution of molecular velocities is not extant then there is a role for a molecular dynamics calculation of the velocities. The highest-speed molecules also are the carriers of the negentropy and so the Gibbs free energy [3,58,59]. Simulation by molecular dynamics of the fat tailed Lévy distributions characteristic of the observed scale invariance and the associated statistical multifractality would be an important step forward.

A further involvement of line shapes is in the shape of the actinic absorptions in the visible and ultraviolet that are responsible for the normal photodissociations that produce free radicals. These line shapes are also important when visible and ultraviolet spectroscopy is used in atmospheric instruments to retrieve and measure atmospheric constituents, such as chlorine dioxide and ozone $[61,62]$.

The isotopic structure of atmospheric molecules [63] has been used to characterise the interplay of radiation, chemical kinetics, cloud physics and transport processes [64]. The interpretation of both the mass-dependent and independent isotope fractionations, and the intramolecular ordering has relied on theoretical chemical results [65]. However, the laboratory experiments and their theoretical interpretation have not been connected to the non-equilibrium state of the atmosphere; for example, the non-thermalisation of the photofragments of ozone photodissociation could affect the distribution and internal ordering of oxygen isotopes in ozone, water, carbon dioxide, nitrous oxide and aerosols via accelerated reaction rates. Isotopic exchange at aerosol surfaces and interiors could be rapid and affected by non-thermal velocities. Molecular dynamics could assess the magnitude of such effects.

\section{Turbulence, Symmetry Breaking, Emergence and Frustration}

The atmosphere is a non-ergodic system, with turbulence emerging as a result of symmetry breaking and the minimisation of Gibbs free energy, with the consequent dilution of energy density $[7,12]$. The panoply of non-linear interactions causes the frustration that sustains the non-ergodicity [66]; for example, there are neither the physical mechanisms nor their rates of operation in the winter polar vortices to make the law of mass action applicable to the observed ozone loss over the entire volume of affected air $[67,68]$. Symmetry breaking is evident in widely varying phenomena such as aerosol fission [48,69-71]; sudden stratospheric warmings splitting the polar vortex [10,11,72]; non-Doppler and non-Lorentzian line shapes; limitation of the Fourier components used in dynamical meteorology; and the emergence of fluid flow when a directional flux breaks the symmetry of a thermalised atomic population [2].

Further examples of symmetry breaking include the asymmetry of the air-water interface [71]; the temperature difference across the terminator [3,12]; the tilt of the planetary axis of rotation; the Coriolis force; the distribution of land and sea-plate tectonics is the ultimate symmetry breaker for the fluid envelope. The Gibbs free energy, in inducing 
the dilution of energy density and organised but turbulent fluid flow, pays the entropy cost by dissipation via infrared radiation to space. That causes the irreversibility and symmetry breaking that underpin the arrow of time and the working of the 2nd Law in the open system far from equilibrium. It is a manifestation of the principle "more is different" [73]. While symmetry breaking is perhaps more familiar on the microscopic scale to theoretical chemists-for example, in the Jahn-Teller effect-the key point made by Anderson [73] is that it is an essential link between the linear quantum domain and the non-linear mesoscopic and macroscopic domains.

In principle, molecular dynamics could provide a bottom-up, upscale representation of turbulence for numerical models, which mainly use equilibrium approaches and downscale parametrisations, which have significant limitations [74]. The driving force for turbulence under the framework of statistical multifractals is argued to be fluxes of energy density [7] and dilution of energy density under the Alder-Wainwright mechanism [5]. Quantum mechanics is linear, while the macroscopic world is non-linear. Fast atoms and molecules break the symmetry of a randomised (thermalised, equilibrated) air sample at the mesoscale, carrying the Gibbs free energy that dilutes energy density and propagates non-linear turbulence via the Alder-Wainwright mechanism.

\section{A Proposed Programme of Theoretical Chemistry Research}

Given the problems and opportunities outlined above, what approach can theoretical chemists take? So far, considerable efforts have been made into particular chemical processes such as photodissociation, isotope effects, aerosol air-water interfaces, rovibrational spectra and chemical kinetics. Since the Navier-Stokes equation for fluid flow was formulated in the mid-19th century, continual efforts to the present have failed to find analytical solutions. With that failure has gone the sought-after mathematical framework for turbulence, and in the present context its representation in numerical models of the atmosphere, for weather forecasting to some extent and more seriously in models seeking to simulate climate [75]. Because air maintains its energy fluxes by molecular processes, particularly absorption and emission of radiation followed by collisional energy transfer, energy must transfer up from the smallest microscopic scales. In the denser, lower atmosphere, there are multiple complicating processes, such as pressure broadening of line shapes including weak transitions in $\mathrm{O}_{2}$ and $\mathrm{N}_{2}$ [60], phase changes of water and cloud physics. The triangular interaction of radiation-fluid mechanics-chemistry is simplest in the upper stratosphere, with low pressures, more Doppler-like line shapes, and dominant photochemistry. It is where the most serious violations of the assumptions used in modelling the flow occur, and where there is a systematic cold bias compared to observations [4]. The dominant aqueous sulphuric acid aerosols boil in the upper stratosphere, a simplification. Accordingly, it is possible to contemplate a molecular dynamics simulation of an air packet in the upper stratosphere yielding ab initio values for interactive fluid mechanics, radiative transfer and chemistry. Such an effort will be extremely demanding from both the scientific and computational points of view, for example deploying Lennard-Jones 12-6 intermolecular potentials rather than billiards [76]; the two viewpoints are intimately linked in this context of course. The inability to parallelise collisional trajectories causes major demands on processing time. The specification of boundary conditions and the limitations of thermal bath assumptions are also likely to be obstacles [76,77]. Isotropic turbulence is not observed in the atmosphere [78]. The scale-free network that is stratospheric chemistry has several free radicals that are central to its operation $[5,79,80]$ and it will be necessary to proceed via piecewise initial essays [76]. This is how consuming and difficult it is to model each of the processes that interact in the atmosphere using molecular dynamics. It will probably be necessary to adopt the simplest formulation that will work for each individual process, and then try pairwise interactions before trying to represent all interactions in the way that climate models do. However, if the programme can be executed successfully, the opportunity to address some major unsolved issues will exist. These include the mesoscale region between the microscopic and macroscopic [9], the understanding of turbulence 
as an emergent property $[4,5,7,12]$ and how to deal with small-scale parametrisation in numerical models $[74,75]$. The standard book for molecular behaviour in gases and liquids has been Hirschfelder, Curtiss and Bird [81], while that by Chapman and Cowling [82] has also been influential. Nevertheless, a convincing formulation of a mesoscopic bridge [9] from microscopic to macroscopic scales has long been missing; theoretical chemists were involved in saying so. An advance driven by theoretical chemists would be fitting. The various molecular processes to be simulated take in assorted aspects of theoretical chemistry. It is best left to the judgement of experts in these fields as to how to proceed. It may take collaborative efforts. Molecular dynamics has been surveyed recently [83].

Will the value of the Hurst exponent $H$ for individual chemical species emerge as an indicator of production $(H>5 / 9)$, loss $(H<5 / 9)$ or inertness $(H=5 / 9)$ ? [3-5,67,68]? Such a success would have wider applicability than the upper stratosphere. The quantitative framing of turbulence has been an elusive goal for two centuries. Theoretical chemistry has the ability to achieve this in principle, by showing that it is an emergent property of fluid mechanics arising from symmetry breaking, driven by the Gibbs free energy of the most energetic molecules acting to minimise energy density.

Funding: There was no funding for this work.

Institutional Review Board Statement: None necessary.

Informed Consent Statement: None necessary.

Data Availability Statement: Data used are available on NASA Airborne Database https: / earthdata. nasa.gov/esds/impact/admg/the-airborne-inventory (accessed on 5 June 2021).

Acknowledgments: Vaida: V. provided a critique of Section 3.

Conflicts of Interest: The author declares no vested interest.

\section{References}

1. Alder, B.J.; Wainwright, T.E. Phase transition for a hard sphere system. J. Chem. Phys. 1957, 27, 1208-1209. [CrossRef]

2. Alder, B.J.; Wainwright, T.E. Decay of the velocity autocorrelation function. Phys. Rev. 1970, 1, 18-21. [CrossRef]

3. Tuck, A.F. Atmospheric Turbulence: A Molecular Dynamics Perspective; Oxford University Press: Oxford, UK, 2008; ISBN 978-0-19-923653-4.

4. Tuck, A.F. From molecules to meteorology via turbulent scale invariance. Q. J. R. Meteorol. Soc. 2010, 136, 1125-1144. [CrossRef]

5. Tuck, A.F. Proposed empirical entropy and Gibbs energy based on observations of scale invariance in open nonequilibrium systems. J. Phys. Chem. A 2017, 121, 6620-6629. [CrossRef]

6. Schertzer, D.; Lovejoy, S. Physical modeling and analysis of rain and clouds by anisotropic scaling multiplicative processes. J. Geophys. Res. D 1987, 92, 9693-9714. [CrossRef]

7. Lovejoy, S.; Schertzer, D. The Weather and Climate: Emergent Laws and Multifractal Cascades; Cambridge University Press: Cambridge, UK, 2013; ISBN 978-1-107-01898-3.

8. Kadau, K.; Barber, J.L.; Germann, T.C.; Holian, B.L.; Alder, B.J. Atomistic methods in fluid simulation. Philos. Trans. R. Soc. A 2012, 368, 1547-1560. [CrossRef] [PubMed]

9. Laughlin, R.B.; Pines, D.; Schmalian, J.; Stojkovic, B.P.; Wolynes, P. The middle way. Proc. Natl. Acad. Sci. USA 2000, 97, 32-37. [CrossRef] [PubMed]

10. O'Neill, A.; Pope, V.D. The coupling between radiation and dynamics in the stratosphere. Adv. Space Res. 1993, 13, 351-358. [CrossRef]

11. O'Neill, A.; Oatley, C.L.; Charlton-Perez, A.J.; Mitchell, D.M.; Jung, T. Vortex splitting on a planetary scale in the stratosphere by cyclogenesis on a sub-planetary scale in the troposphere. Q. J. R. Meteorol. Soc. 2017, 143, 691-705. [CrossRef]

12. Tuck, A.F. Entropy and Exergy in Renewable Energy; Chapter NN, Scale Invariant Turbulence and Gibbs Free Energy in the Atmosphere; Wang, L.-S., Ed.; IntechOpen: London, UK, 2020. [CrossRef]

13. Middlebrook, A.M.; Murphy, D.M.; Thomson, D.S. Observation of organic material in individual marine particles at Cape Grim during the First Aerosol Characterization Experiment (ACE-1). J. Geophys. Res. D 1998, 103, 16475-16483. [CrossRef]

14. Murphy, D.M.; Thomson, D.S.; Mahoney, M.J. In situ measurements of organics, meteoritic material, mercury and other elements in aerosols from 5 to 19 kilometers. Science 1998, 282, 1664-1669. [CrossRef]

15. Gard, E.E.; Kleeman, M.J.; Gross, D.S.; Hughes, L.S.; Allen, J.O.; Morrical, B.D.; Fergenson, D.P.; Dienes, T.; Gälli, M.E.; Johnson, R.J.; et al. Direct observation of heterogeneous chemistry in the atmosphere. Science 1998, 279, 1184-1187. [CrossRef] [PubMed]

16. Ellison, G.B.; Tuck, A.F.; Vaida, V. Atmospheric processing of organic aerosols. J. Geophys. Res. D 1999, 104, 1163-11641. [CrossRef] 
17. Vaida, V.; Kjaergaard, H.G.; Hintze, P.E.; Donaldson, D.J. Photolysis of sulfuric acid vapor by visible solar radiation. Science 2003, 299, 1566-1568. [CrossRef] [PubMed]

18. Miller, Y.; Gerber, R.B. Dynamics of vibrational overtone excitations of $\mathrm{H}_{2} \mathrm{SO}_{4}, \mathrm{H}_{2} \mathrm{SO}_{4}$. $\mathrm{H}_{2} \mathrm{O}$ : Hydrogen-hopping and photodissociation processes. J. Am. Chem. Soc. 2006, 128, 9594-9595. [CrossRef]

19. Donaldson, D.J.; Vaida, V. The influence of organic films at the air-aqueous boundary on atmospheric processes. Chem. Revs. 2006, 106, 1445-1461. [CrossRef]

20. Miller, Y.; Gerber, R.B.; Vaida, V. Photodissociation yields for vibrationally excited states of sulfuric acid under atmospheric conditions. Geophys. Res. Lett. 2007, 34, L16820. [CrossRef]

21. Takahashi, K.; Plath, K.L.; Skodje, R.T.; Vaida, V. Dynamics of vibrational overtone excited pyruvic acid in the gas phase: Line broadening through hydrogen-atom chattering. J. Phys. Chem. A 2008, 112, 7321-7331. [CrossRef] [PubMed]

22. Maroń, M.K.; Takahashi, K.; Shoemaker, R.K.; Vaida, V. Hydration of pyruvic acid to its geminal-diol, 2, 2-dihydroxypropanoic acid, in a water-restricted environment. Chem. Phys. Lett. 2011, 513, 184-190. [CrossRef]

23. Kramer, Z.C.; Takahashi, K.; Vaida, V.; Skodje, R.T. Will water act as a photocatalyst for cluster phase chemical reactions? Vibrational overtone-induced dehydration reaction of methanediol. J. Chem. Phys. 2012, 136, 164302. [CrossRef]

24. Griffith, E.C.; Carpenter, B.K.; Shoemaker, R.K.; Vaida, V. Photochemistry of aqueous pyruvic acid. Proc. Natl. Acad. Sci. USA 2013, 110, 11714-11719. [CrossRef]

25. Griffith, E.C.; Rapf, R.J.; Shoemaker, R.K.; Carpenter, B.K.; Vaida, V. Photoinitiated Synthesis of Self-Assembled Vesicles. J. Am. Chem. Soc. 2014, 136, 3784-3787. [CrossRef] [PubMed]

26. Griffith, E.C.; Perkins, R.J.; Telesford, D.M.; Adams, E.M.; Cwiklik, L.; Allen, H.C.; Roeselova, M.; Vaida, V. Interaction of L-Phenylalanine with a Phospholipid Monolayer at the Water-Air Interface. J. Phys. Chem. B 2015, 119, 9038-9048. [CrossRef] [PubMed]

27. Perkins, R.J.; Shoemaker, R.K.; Carpenter, B.K.; Vaida, V. Chemical Equilibria and Kinetics in Aqueous Solutions of Zymonic Acid. J. Phys. Chem. A 2016, 120, 10096-10107. [CrossRef]

28. Reed Harris, A.E.; Doussin, J.F.; Carpenter, B.K.; Vaida, V. Gas-Phase Photolysis of Pyruvic Acid: The Effect of Pressure on Reaction Rates and Products. J. Phys. Chem. A 2016, 120, 10123-10133. [CrossRef]

29. Perkins, R.J.; Kukharchuk, A.; Delcroix, P.; Shoemaker, R.K.; Roeselova, M.; Cwiklik, L.; Vaida, V. The Partitioning of Small Aromatic Molecules to Air-Water and Phospholipid Interfaces Mediated by Non-Hydrophobic Interactions. J. Phys. Chem. B 2016, 120, 7408-7422. [CrossRef] [PubMed]

30. Rapf, R.J.; Perkins, R.J.; Carpenter, B.K.; Vaida, V. Mechanistic Description of Photochemical Oligomer Formation from Aqueous Pyruvic Acid. J. Phys. Chem. A 2017, 121, 4272-4282. [CrossRef] [PubMed]

31. Rapf, R.J.; Perkins, R.J.; Yang, H.S.; Miyake, G.; Carpenter, B.K.; Vaida, V. Photochemical Synthesis of Oligomeric Amphiphiles from Alkyl Oxoacids in Aqueous Environments. J. Am. Chem. Soc. 2017, 139, 6946-6959. [CrossRef] [PubMed]

32. Gordon, B.P.; Moore, F.G.; Scatena, L.F.; Richmond, G.L. On the rise: Experimental and computational vibrational sum frequency spectroscopy studies of pyruvic acid and its surface-active oligomer species at the air-water interface. J. Phys. Chem. A 2019, 123, 10609-10619. [CrossRef]

33. Church, J.R.; Vaida, V.; Skodje, R.T. Gas-Phase Reaction Kinetics of Pyruvic Acid with OH Radicals: The Role of Tunneling, Complex Formation, and Conformational Structure. J. Phys. Chem. A 2020, 124, 790-800. [CrossRef]

34. Church, J.R.; Vaida, V.; Skodje, R.T. Kinetic study of gas-phase reactions of pyruvic acid with $\mathrm{HO}_{2}$. J. Phys. Chem A 2021, 125, 2232-2242. [CrossRef]

35. Kappes, K.J.; Deal, A.M.; Jesperson, M.F.; Blair, S.L.; Doussin, J.-F.; Cazaunau, M.; Pangui, E.; Hopper, B.N.; Johnson, M.S.; Vaida, V. Chemistry and photochemistry of pyruvic acid at the air-water interface. J. Phys. Chem. A 2021, 125, 1036-1049. [CrossRef] [PubMed]

36. Gertner, B.J.; Hynes, J.T. Model molecular dynamics simulation of hydrochloric acid ionization at the surface of stratospheric ice. Faraday Discuss. 1998, 110, 301-322. [CrossRef]

37. Reyes, J.Y.; Nagy, T.; Meuwly, M. Competitive reaction pathways in vibrationally induced photodissociation of $\mathrm{H}_{2} \mathrm{SO}_{4}$. Phys. Chem. Chem. Phys. 2014, 34, 18533-18544. [CrossRef]

38. Ruiz-Lopez, M.F.; Francisco, J.S.; Martins-Costa, M.T.C.; Anglada, J.M. Molecular reactions at aqueous interfaces. Nat. Rev. Chem. 2020, 4, 459-475. [CrossRef]

39. Tuck, A.F.; Donaldson, D.J.; Hitchman, M.H.; Richard, E.C.; Tervahattu, H.; Vaida, V.; Wilson, J.C. On geoengineering with sulphate aerosols in the tropical upper troposphere and lower stratosphere. Clim. Chang. 2008, 90, 315-331. [CrossRef]

40. Tuck, A.F. Gibbs free energy and reaction rate acceleration in and on microdroplets. Entropy 2019, 21, 1044. [CrossRef]

41. Cooks, R.G.; Ouyang, Z.; Takats, Z.; Wiseman, J.M. Ambient mass spectrometry. Science 2006, 311, 1560-1570. [CrossRef]

42. Lee, J.K.; Banerjee, S.; Nam, H.G.; Zare, R.N. Acceleration of reaction in charged microdroplets. Q. Rev. Biophys. 2015, 48, 437-444. [CrossRef] [PubMed]

43. Marsh, B.M.; Iyer, K.; Cooks, R.G. Reaction Acceleration in Electrospray Droplets: Size, Distance, and Surfactant Effects. J. Am. Soc. Mass Spectrom. 2019, 30, 2022-2030. [CrossRef] [PubMed]

44. Xiong, H.; Lee, J.K.; Zare, R.N.; Min, W. Strong Electric Field Observed at the Interface of Aqueous Microdroplets. J. Phys. Chem. Lett. 2020, 11, 7423-7428. [CrossRef] 
45. Vaida, V. Prebiotic phosphorylation enabled by microdroplets. Proc. Natl. Acad. Sci. USA 2017, 114, 12359-12361. [CrossRef] [PubMed]

46. Wilson, K.R.; Prophet, A.M.; Rovelli, G.; Willis, M.D.; Rapf, R.J.; Jacobs, M.I. A kinetic description of how interfaces accelerate reactions in micro-compartments. Chem. Sci. 2020, 11, 8533-8545. [CrossRef]

47. Dobson, C.M.; Ellison, G.B.; Tuck, A.F.; Vaida, V. Atmospheric aerosols as prebiotic chemical reactors. Proc. Natl. Acad. Sci. USA 2000, 97, 11864-11868. [CrossRef] [PubMed]

48. Donaldson, D.J.; Tuck, A.F.; Vaida, V. The asymmetry of organic aerosol fission and prebiotic chemistry. Orig. Life Evol. B 2002, 32, 237-245. [CrossRef]

49. Griffith, E.C.; Tuck, A.F.; Vaida, V. Ocean-Atmosphere Interactions in the Emergence of Complexity in Simple Chemical Systems. Accounts Chem. Res. 2012, 45, 2106-2113. [CrossRef]

50. Tuck, A. The role of atmospheric aerosols in the origin of life. Surv. Geophys. 2002, 23, 379-409. [CrossRef]

51. Strow, L.L.; Tobin, D.C.; McMillan, W.W.; Hannon, S.E.; Smith, W.L.; Revercomb, H.E.; Knuteson, R.O. Impact of a new water vapor continuum and lineshape model on observed high resolution infrared radiances. J. Quant. Spectr. Rad. Transfer 1998, 59, 303-317. [CrossRef]

52. Menzel, W.P.; Tobin, D.C.; Revercomb, H.E. Infrared remote sensing with meteorological satellites. Adv. Atomic Molec. Phys. 2016, 65, 193-264.

53. Mlawer, E.J.; Payne, V.H.; Moncet, J.-L.; Delamere, J.S.; Alvarado, M.J.; Tobin, D.C. Development and recent evaluation of the MT_CKD model of continuum absorption. Phil. Trans. R. Soc. A 2012, 370, 2520-2556. [CrossRef]

54. Vaida, V.; Daniel, J.S.; Kjaergaard, H.G.; Goss, L.M.; Tuck, A.F. Atmospheric absorption of near infrared and visible solar radiation by the hydrogen bonded water dimer. Q. J. R. Meteorol. Soc. 2001, 127, 1627-1643. [CrossRef]

55. Evans, G.T.; Vaida, V. Aggregation of water molecules: Atmospheric implications. J. Chem. Phys. 2000, 113, 6652-6659. [CrossRef]

56. Vaida, V. Perspective: Water cluster mediated atmospheric chemistry. J. Chem. Phys. 2011, 135, 020901. [CrossRef] [PubMed]

57. Donaldson, D.J.; Frost, G.J.; Rosenlof, K.H.; Tuck, A.F.; Vaida, V. Atmospheric radical production by excitation of vibrational overtones via absorption of visible light. Geophys. Res. Lett. 1997, 24, 2651-2654. [CrossRef]

58. Takahashi, K.; Taniguchi, N.; Sato, Y.; Matsumi, Y. Nonthermal steady state translational energy distributions of $\mathrm{O}\left({ }^{1} \mathrm{D}\right)$ atoms in the stratosphere. J. Geophys. Res. D 2002, 107, 4290. [CrossRef]

59. Baloïtcha, E.; Balint-Kurti, G.G. The theory of the photodissociation of ozone in the Hartley continuum: Effect of vibrational excitation and $\mathrm{O}\left({ }^{1} \mathrm{D}\right)$ atom velocity distribution. Phys. Chem. Chem. Phys. 2005, 7, 3829-3833. [CrossRef] [PubMed]

60. Goody, R.M.; Yung, Y.L. Atmospheric Radiation: Theoretical Basis, 2nd ed.; Oxford University Press: New York, NY, USA, 1989; ISBN 0-19-505134-3.

61. Richard, E.C.; Vaida, V. The photochemical dynamics of the $\tilde{\mathrm{A}}^{2} \mathrm{~A}_{2}$ state of chlorine dioxide. J. Chem. Phys. 1991, $94,163-171$. [CrossRef]

62. Axson, J.L.; Washenfelder, R.A.; Kahan, T.F.; Young, C.J.; Vaida, V.; Brown, S.S. Absolute ozone absorption cross section in the Huggins Chappuis minimum (350-470 nm) at 296 K. Atmos. Chem. Phys. 2011, 11, 11581-111590. [CrossRef]

63. Thiemens, M.H. Mass-independent isotope fractionations and their applications. ACS Symp. Ser. 1992, 502, 138-154.

64. Yeung, L.Y.; Murray, L.T.; Ash, J.L.; Young, E.D.; Boering, K.A.; Atlas, E.L.; Schauffler, S.M.; Lueb, R.A.; Langenfelds, R.L.; Krummel, P.B.; et al. Isotopic ordering in atmospheric $\mathrm{O}_{2}$ as a tracer of ozone photochemistry and the tropical atmosphere. J. Geophys. Res. D 2016, 121, 12541-12559.

65. Marcus, R.A. Strange and unconventional isotope effects in ozone formation. Science 2001, 293, $259-263$.

66. Wolf, Y.I.; Katsnelson, M.I.; Koonin, E.V. Physical foundations of biological complexity. Proc. Natl. Acad. Sci. USA 2018, 115, E8678-E8687. [CrossRef]

67. Tuck, A.F.; Hovde, S.J.; Richard, E.C.; Fahey, D.W.; Gao, R.-S. A scaling analysis of ER-2 data in the inner vortex during January-March 2000. J. Geophys. Res. D 2002, 108, 8306. [CrossRef]

68. Tuck, A.F.; Gao, R.-S.; Richard, E.C. Law of mass action in the Arctic lower-stratospheric polar night vortex January-March 2000: $\mathrm{ClO}$ scaling and the calculation of ozone loss rates in a turbulent fractal medium. J. Geophys. Res. D 2003, 108, 4451. [CrossRef]

69. Donaldson, D.J.; Tuck, A.F.; Vaida, V. Spontaneous fission of atmospheric aerosol particles. Phys. Chem. Chem. Phys. 2001, 3, 5270-5273. [CrossRef]

70. Donaldson, D.J.; Tervahattu, H.; Tuck, A.F.; Vaida, V. Organic aerosols and the origin of life: An hypothesis. Orig. Life Evol. Biosph. 2004, 34, 57-67. [CrossRef]

71. Deal, A.M.; Rapf, R.J.; Vaida, V. Water-air interfaces as environments to address the water paradox in prebiotic chemistry; a physical chemistry perspective. J. Phys. Chem A 2021, in press. [CrossRef]

72. Tuck, A.F.; Hovde, S.J.; Bui, T.P. Scale invariance in jet streams: ER-2 data around the lower-stratospheric polar night vortex. Q. J. R. Meteorol. Soc. 2004, 130, 2423-2444. [CrossRef]

73. Anderson, P.W. More is different-broken symmetry and the nature of the hierarchical structure of science. Science 1972, 177, 393-396. [CrossRef]

74. Palmer, T.N. A nonlinear perspective on model error: A proposal for non-local stochastic-dynamical parameterization in weather and climate prediction models. Q. J. R. Meteorol. Soc. 2001, 127, 279-304. [CrossRef]

75. Lovejoy, S. Weather, Macroweather and the Climate; Oxford University Press: Oxford, UK, 2019; ISBN 978-0-19-086421-7.

76. Rapaport, D.C. The Art of Molecular Dynamics Simulation, 2nd ed.; Cambridge University Press: Cambridge, UK, 2004. 
77. Evans, D.J.; Williams, S.R.; Searles, D.J. On the entropy of relaxing deterministic systems. J. Chem. Phys. 2011, $135,194107$. [CrossRef]

78. Lovejoy, S.; Tuck, A.F.; Hovde, S.; Schertzer, D. Is isotropic turbulence relevant in the stratosphere? Geophys. Res. Lett. 2007, 34, L15802. [CrossRef]

79. Solé, R.V.; Munteanu, A. The large-scale organization of chemical reaction networks in astrophysics. Europhys. Lett. 2004, 68, 170-176. [CrossRef]

80. Tuck, A.F. Perspective on aircraft in the stratosphere: 50 years from COMESA through the ozone hole to climate. Q. J. R. Meteorol. Soc. 2021, 147, 713-727. [CrossRef]

81. Hirschfelder, J.O.; Curtiss, C.F.; Bird, R.B. Molecular Theory of Gases and Liquids, 2nd ed.; Wiley: New York, NY, USA, 1964.

82. Chapman, S.; Cowling, T.G. The Mathematical Theory of Non-Uniform Gases, 3rd ed.; Cambridge University Press: Cambridge, UK, 1970.

83. Brooks, C.L.; Case, D.A.; Plimpton, S.; Rouse, B.; van der Spoel, D.; Tajkhorshid, E. Classical molecular dynamics. J. Chem. Phys. 2021, 154, 100401. [CrossRef] [PubMed] 